\title{
Defining the global health system and systematically mapping its network of actors
}

Steven J. Hoffman ${ }^{1,2,3^{*}}$ and Clarke B. Cole C $^{1,4}$

\begin{abstract}
Background: The global health system has faced significant expansion over the past few decades, including continued increase in both the number and diversity of actors operating within it. However, without a stronger understanding of what the global health system encompasses, coordination of actors and resources to address today's global health challenges will not be possible.

Methods: This study presents a conceptually sound and operational definition of the global health system. Importantly, this definition can be applied in practice to facilitate analysis of the system. The study tested the analytical helpfulness of this definition through a network mapping exercise, whereby the interconnected nature of websites representing actors in the global health system was studied.

Results: Using a systematic methodology and related search functions, 203 global health actors were identified, representing the largest and most transparent list of its kind to date. Identified global health actors were characterized and the structure of their social network revealed intriguing patterns in relationships among actors.

Conclusions: These findings provide a foundation for future inquiries into the global health system's structure and dynamics that are critical if we are to better coordinate system activities and ensure successful response to our most pressing global health challenges.
\end{abstract}

Keywords: Global Health, Internet, Search engine, World Health Organization

\section{Background}

The global health system has faced significant expansion over the past few decades, including continued increase in both the number and diversity of actors within it [1-5]. Recognition is growing that better coordination of these actors is necessary if we are to ensure effective response to the most pressing contemporary global health challenges. However, the research literature demonstrates little agreement over definitions of relevant concepts and their practical applications [6]. Without a stronger empirical understanding of what the global health system actually encompasses - including its components, how they

\footnotetext{
* Correspondence: steven.hoffman@globalstrategylab.org

${ }^{1}$ Global Strategy Lab, Dahdaleh Institute for Global Health Research, Faculty of Health and Osgoode Hall Law School, York University, 4700 Keele Street, Toronto, Ontario M3J 1P3, Canada

${ }^{2}$ Department of Health Research Methods, Evidence \& Impact and McMaster Health Forum, McMaster University, Hamilton, Ontario, Canada

Full list of author information is available at the end of the article
}

operate, and how well they do so - such coordination will be more difficult.

In particular, there is a lack of understanding over which actors should actually be considered as part of the global health system. The range of actors included is frequently cited at "more than 40 bilateral donors, 26 UN agencies, 20 global and regional funds and 90 global health initiatives" [7]. However, given the continued expansion of the system, this 2007 statistic is outdated and information is also lacking on how it was derived and which actors it includes. In order to develop an updated account of global health actors, we must find a meaningful way to map them, considering not only their existence but also the ways in which they interact with one another. Online networks - constructed by realworld actors - present a unique solution to this challenge. Coscia, Hausmann \& Hidalgo (2013) used online information to study the structure of international aid 
coordination, creating and mapping a network of donor organizations, recipient countries, and development issues [8]. Similarly, Coscia \& Rios (2012) developed and validated a framework that used Internet content, such as online newspapers and blogs, to reveal areas of operation of Mexican drug trafficking organizations [9]. These studies demonstrated the potential to use online networks for global health system analysis. Further studies using offline data demonstrate the value of mapping social networks within the health sector. For example, Bowen et al. (2014) applied social network analysis to identify key organizations engaged in developing health-related climate change adaptation activities in Cambodia [10].

This study aims to advance our understanding of the global health system by answering two questions. First, what is the 'global health system'? And second, who populates this system? While many answers are possible, we limit ourselves to definitions that can be operationalized with inclusion/exclusion criteria and mapping methods that are systematic, transparent, and replicable. In this way, we produce work that is hopefully analytically useful, minimally biased, and foundational for future inquiries.

In answering these two questions, we first present an operational definition of the global health system that sets clear boundaries and can actually be applied to map global health actors and their relationships. Second, we used online network relationships to generate a list of 203 global health actors and characterize those actors; in effect, using the online network of global health actors to better understand the offline global health system [11].

\section{Methods}

\section{Defining the global health system}

Relevant literature from global health, international relations, law, political science, and public policy was reviewed to understand how scholars are conceptualizing the global health system and how cognate global systems have previously been defined. This literature was used to inform development of a clear, applicable definition of the global health system (see Additional file 1 for a more detailed account of this process).

\section{Mapping the global health system}

The helpfulness of this definition was tested through application to a mapping exercise of the global health system, in which websites that represent global health actors were identified. Using a systematic search protocol, a network of global health actors was created, characterized, and its social network analyzed. This methodology took advantage of the Internet's network structure in a novel way, mapping online interactions to probabilistically identify key offline relationships in the global health system.
Importantly, the methodology was designed to be internally valid, transparent, and replicable, identifying a minimally-biased network of actors operating within the global health system. It was not designed to create a comprehensive or complete list of all actors operating within the global health system or to identify a list of actors based on their power or influence within the system. Through this process, it is hoped that our study output will provide a snapshot of the current global health architecture, reflecting a true network that can be used to both inform our understanding of the global health system and allow for further application and development of our methodology.

\section{Data mining}

A systematic search of the Internet was conducted to identify the online network of global health actors. This involved the use of a related search function that can identify websites that represent global health actors. Related search functions use algorithms - different combinations of connectivity analysis, content analysis and page usage - to identify web pages that are topically similar but not identical to one another. Web connectivity algorithms, such as co-citation analysis, exploit the hyperlink-structure of the Internet to find web pages that reference each other. They work under the basic assumption that web pages connected by hyperlinks contain related content and, if the pages have distinct authors, this linkage suggests the creator of one website found the content of referenced web pages to be valuable. Content analysis algorithms evaluate similarities in topical content found across web pages. This means that web pages with information on similar topics will be considered related. Page usage relates to information gained about a web page when search engine users select a particular link following a given query (e.g., if a user searches for 'university', and selects the search result 'Harvard', it logically follows that Harvard is likely a university) $[12,13]$.

Accordingly, this methodology assumes a website can serve as a minimum criterion for including an actor in a preliminary list of global health actors, as most actors with a capacity to influence global health will, at a minimum, have an online presence. A related search function was used to identify global health actors under the assumption that actors operating offline in the global health system are likely to reference other actors they consider relevant and valuable through linkages to those actors' websites on their own website. It also assumes global health actors are likely to include similar topical content on their websites, and that Internet users are likely to follow similar search and retrieval patterns in accessing the sites of different global health actors. 
Searches were conducted using the publicly available Google search engine (www.google.com) and its corresponding crawler, Googlebot, which maintains a realtime index of over 100 million gigabytes [14]. Searches used the "related:URL" query refinement for which the user enters a specific known URL to find websites that link to it, share similar content and attract the same users [15]. A proxy server was used to search anonymously from a United States-based (U.S.) IP address, and all browser history, caches, and cookies were cleared to prevent personalized results. A pilot test was conducted to refine the systematic search methodology and inclusion/exclusion criteria (detailed more thoroughly in Additional file 2).

A snowball sampling search process was started with the World Health Organization's (WHO's) website, thereby placing this United Nations (UN) agency at the centre of our global health system mapping [3, 4]. This was done by conducting a related search using its website (i.e., "related:www.who.int"). Next, in a second-stage search, related searches were conducted on all eligible websites identified in the original search. Results were extracted for eligibility screening.

\section{Data screening}

Websites retrieved through the review were included in our mapping of the global health system if they met the following three criteria, based on our definition for a global health actor (see Additional files 3 and 4 for detailed screening forms):

1. The result represents an individual or organization (i.e., an actor).

2. The actor operates in three or more countries (i.e., transnationally).

3. The actor identifies improving health as one of its primary intents (i.e., health focus).

The inclusion/exclusion criteria were applied in two stages. In the first stage, website summaries available in search results were reviewed to determine whether the sites met at least two of the criteria. Those websites that passed stage 1 screening were then reviewed in full to determine whether they met all three criteria. If so, they were included in the final mapping.

Specifically, for the full website review, the "About" web page (or its equivalent) of each retrieved website was reviewed for its ability to meet all three inclusion criteria and eligible websites were included as global health actors in the systematic review. All uncertainties were reviewed and, where necessary, additional web pages on an actor's website were accessed for further information. Identified parent organizations were evaluated for inclusion in the review. If eligible, corresponding child organizations were omitted (see Fig. 1 for a flowchart of the review process). A parent organization is the umbrella organization in cases where there are multiple arms or affiliates as part of a single organization. A child organization is one autonomous section or offshoot of a parent organization. For example, CARE International is a parent organization and CARE Canada is one of its child organizations. If both organizations met the inclusion criteria during the review, CARE International would be included in the final review and CARE Canada would be omitted.

\section{Data analysis}

Findings from the systematic review were entered into a database of actors in the global health system. To characterize each global health actor, additional data were extracted from its website. Global health actors were categorized according to primary types of entities in the global health system as outlined in Frenk \& Moon (2013) [3]: headquarters location, year of inception, and whether the primary intent of the organization was to improve health (as opposed to whether promoting health was just one of several primary objectives). Actors were placed into one distinct category under each variable.

The network was modelled to allow visualization of the global health system and facilitate observations of the network structure. The network was constructed such that each global health actor was assigned a node and characteristics of each respective actor were recorded as node attributes. Directed edges were used to indicate each time a particular actor linked to another actor in the network (i.e., each time the "related:URL" search for a global health actor's website yielded a result that was also an actor). All duplicate results were included such that a directed edge exists in the network to indicate every time an actor appeared in any set of "related:URL" search results throughout the review. Gephi 0.8.2 beta was used to construct and analyze the network [16].

According to social network theory, actors gain power within social networks by holding advantageous positions relative to other actors. Centrality, one of the most studied metrics in social network analysis, allows an actor's position in its network to be described relative to others'. Three relatively simple centrality metrics that are important and popularly used were applied in this analysis: degree centrality, betweenness centrality and closeness centrality $[17,18]$.

Nodes with high degree centrality are connected to a large number of other nodes in the network. They have a high probability of receiving and transmitting information that flows through the network, and are therefore considered highly active players. Betweenness centrality measures how often a node appears within the shortest 


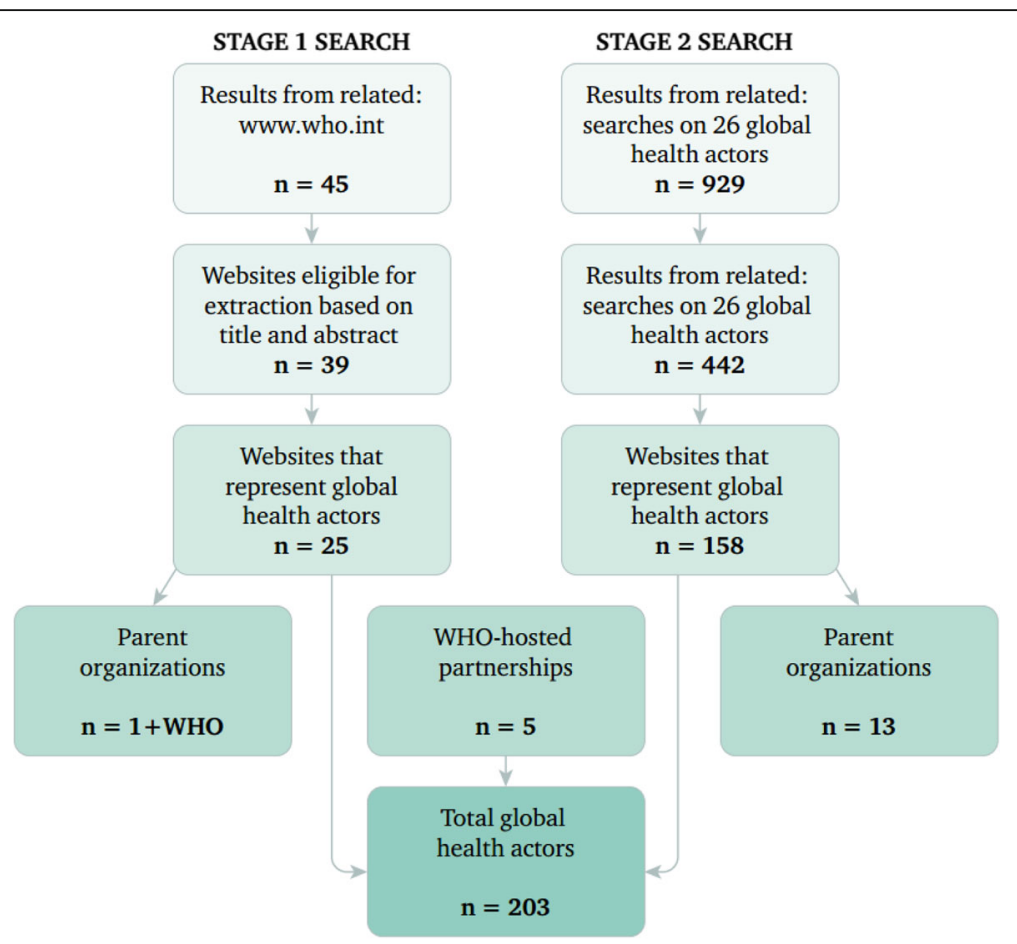

Fig. 1 Flowchart of the systematic review

path between nodes. It describes an actor's level of control over information in the network, and as such, actors with high betweenness centrality function as information brokers in the network relied upon to communicate and enhance collaboration between sub-communities. Closeness centrality measures the degree to which a node is close to all others in the network. Nodes that are central by closeness can reach most or all others in the network on average in fewer steps than others. They are described as being able to communicate with high efficiency in terms of time and cost. Analysis of the global heath network using these three metrics allows global health actors with three basic sources of advantage in the network to be identified: advantage based on communication activity, control of information, and independence and efficiency, respectively $[17,18]$.

\section{Validation exercise}

The findings of this study were presented to senior leaders of seven prominent global health organizations (i.e., Gavi, the Vaccine Alliance, Global Fund to Fight AIDS, Tuberculosis and Malaria, Joint United Nations Programme on HIV/AIDS [UNAIDS], United Nations Population Fund, United Nations Children's Fund [UNICEF], WHO and World Bank) at a workshop in Geneva, Switzerland in December 2014 to assess the analytic helpfulness and comprehensiveness of the findings.

\section{Results}

Defining the global health system

The literature review identified an abundance of definitions for key terms related to the global health system (see Additional file 1 for detailed methodology and findings of the literature review). Terms were defined using a variety of approaches and with varying boundaries, highlighting the usefulness of conceptualizing the global health system in different ways to serve different purposes [3].

Building on the work of Slzezak et al. (2010), [4] Hoffman et al. (2012), [19] and Frenk \& Moon (2013), [3] the following definition for the global health system is proposed:

The global health system includes the transnational actors that have a primary intent to improve health and the polylateral arrangements for governance, finance, and delivery within which these actors operate.

Under this definition, the interactions between global health actors are influenced by the actors themselves, the internal arrangements of the system, and external forces, such as actors and arrangements from other important global policy domains. Accordingly, a global health actor is defined as an individual or organization that operates transnationally with a primary intent to improve health (see Table 1 for further explanation of key terms found within this definition). 
Table 1 Global health system definition

Transnational actors: Individuals or organizations that operate in a way that transcends national political borders. Unlike the term international, which may require actors to be stationed in multiple countries, transnational actors may be stationed in only one country so long as they operate across borders.

Polylateral: The interactions among, and governance of, states and non-state actors, which includes interactions between states, between non-state actors, and between states and non-state actors [26]. While the term bilateral concerns relations between two states and the term multilateral concerns relations between three or more states, the term polylateral is more inclusive in that it also considers interactions of non-state entities [26].

\section{Global health system arrangements include: [27]}

Delivery arrangements: relate to how health services are delivered, accessed and catered to meet local priorities, and focus on factors that determine how care is designed to meet consumers' needs, by whom care is provided, where care is provided and with the supports used to those providing and receiving care.

Financial arrangements: relate to how finances flow through health systems, and focus on how systems are financed, types of funding organizations, how to remunerate providers, how products and services are purchased and the incentive structures for consumers.

Governance arrangements: relate to how a health system is governed, and focus on issues such as policy authority, organizational authority, commercial authority, professional authority and about how

stakeholders are involved in health systems decisions and on what terms.

The definition developed is holistic in nature, whereby the system is viewed from a global perspective. As a result, emphasis is placed on transnational actors engaged in such matters as protecting health security, promoting human rights, responding to humanitarian crises, and facilitating international development, and not on national actors or any particular sub-system of actors. The definition is comprehensive in its ability to consider multiple aspects of the system. First, the definition is inclusive of actors that operate with a primary intent to improve health, not limiting the system to include only those actors that hold powerful or influential roles in the global health system. Additionally, the definition considers relations between actors, arrangements that influence the system's functions, as well as the interaction of the system components with internal and external forces. Importantly, the definition also applies to developing our practical understanding of the global health system. The ability to effectively translate conceptual aspects of the definition into clear inclusion/exclusion criteria for a systematic review process, as outlined in this study, illustrates is value at the operational level (refer to Additional files 3 and 4 for detailed application of the definition to inclusion/exclusion screening criteria).

\section{Mapping the global health system Related search results}

A first-stage search for "related:www.who.int" was conducted on March 14, 2014, yielding 45 actors. In total,
25 of these actors plus one parent organization met the inclusion criteria to be considered global health actors. Subsequently, the home page URLs of each of these actors' websites were used in a second-stage "related:URL" search on March 17, 2014. The one exception was the "related:plan-international.org" search, which was conducted on March 24, 2014. A total of 26 independent second-stage searches were conducted and 929 results retrieved. 572 unique results were reviewed. 158 websites, plus those of 13 parent organizations, met the inclusion criteria in this second-stage search to be considered representative of global health actors.

In addition, five WHO-hosted partnerships were included as global health actors [20]. Four of these WHOhosted partnerships would have been impossible to find through our Internet-based network mapping given their websites are hosted on WHO's website. These include the Alliance for Health Policy and Systems Research, European Observatory on Health Systems \& Policies, Global Health Workforce Alliance, and Partnership for Maternal, Newborn \& Child Health. The International Drug Purchase Facility (UNITAID) was also included because it was the only remaining WHO-hosted partnership as listed in provisional agenda item 11.4 of the 134th session of the WHO Executive Board, Hosted Health Partnerships, which neither has a WHO-hosted website nor was identified in the "related:URL" Internet searches.

When including WHO as the seed global health actor, in total we identified 203 global health actors (see Table 2 for a list of global health actors, their corresponding websites and data on selected characteristics).

\section{Social network analysis}

The social network included 198 nodes, representing all global health actors identified through "related:URL" searches, and 412 edges. This does not include the five additional WHO-hosted partnerships that bring the count of global health actors to 203, as they were not identified using the related search function and therefore are not a formal part of the identified online network structure. The social network showed an average of 2.081 connections per actor and a network diameter of 4 . See Table 3 for rankings of the top ten global health actors by degree, betweenness centrality, and closeness centrality. Global health actors' characteristics and centrality scores were used to explore the structure of the network.

\section{Type of entity}

The majority of identified global health actors were global civil society organizations and non-governmental organizations $(n=138)$, followed by public-private partnerships $(n=18)$, professional associations $(n=16)$, UN entities and intergovernmental organizations $(n=11)$, national governments $(n=7)$, private industry $(n=6)$, 
Table 2 List of global health actors included in the systematic review

\begin{tabular}{|c|c|c|c|c|c|c|c|c|}
\hline \multicolumn{2}{|c|}{ Actor } & \multirow{2}{*}{$\begin{array}{l}\text { Code } \\
\text { ABA }\end{array}$} & \multirow{2}{*}{$\begin{array}{l}\text { URL } \\
\text { abtassociates.com }\end{array}$} & \multirow{2}{*}{$\begin{array}{l}\text { Type } \\
7\end{array}$} & \multicolumn{2}{|c|}{ Headquarters Location } & \multirow{2}{*}{$\begin{array}{l}\text { Health as } \\
\text { primary } \\
\text { intent? } \\
\text { No }\end{array}$} & \multirow{2}{*}{$\begin{array}{l}\begin{array}{l}\text { Year of } \\
\text { Inception }\end{array} \\
1965\end{array}$} \\
\hline 1 & Abt Associates & & & & Cambridge, MA & USA & & \\
\hline 2 & Accordia Global Health Foundation & AGH & accordiafoundation.org & 6 & Washington, DC & USA & Yes & 2000 \\
\hline 3 & $\begin{array}{l}\text { ACTION (Global Health Advocacy } \\
\text { Partnership) }\end{array}$ & $\mathrm{ACT}$ & action.org & 6 & Washington, DC & USA & Yes & 2004 \\
\hline 4 & Action Against Hunger International & $\mathrm{AAH}$ & actionagainsthunger.org & 6 & New York City, NY & USA & Yes & 1979 \\
\hline 5 & Action on Smoking and Health & ASH & ash.org & 6 & Washington, DC & USA & Yes & 1967 \\
\hline 6 & Advocates for Youth & AFY & advocatesforyouth.org & 6 & Washington, DC & USA & Yes & 1980 \\
\hline 7 & Aeras & AER & aeras.org & 6 & Rockville, MD & USA & Yes & 2003 \\
\hline 8 & Africa Fighting Malaria & AFM & fightingmalaria.org & 6 & Durban & South Africa & Yes & 2000 \\
\hline 9 & African Leaders Malaria Alliance & ALM & alma2015.org & 2 & New York City, NY & USA & Yes & 2009 \\
\hline 10 & Africare & AFR & africare.org & 6 & Washington, DC & USA & No & 1970 \\
\hline 11 & Against Malaria Foundation & AMF & againstmalaria.com & 6 & St. Albans & UK & Yes & 2004 \\
\hline 12 & AIDS Healthcare Foundation & $\mathrm{AHF}$ & aidshealth.org & 6 & Amsterdam & Netherlands & Yes & 1987 \\
\hline 13 & $\begin{array}{l}\text { Alliance for Health Policy and } \\
\text { Systems Research }\end{array}$ & $\mathrm{AHP}$ & who.int/alliance-hpsr & 4 & Geneva & Switzerland & Yes & 1999 \\
\hline 14 & $\begin{array}{l}\text { America Association of } \\
\text { Occupational Health Nurses }\end{array}$ & AAO & aaohn.org & 8 & Pensacola, FL & USA & No & 1998 \\
\hline 15 & $\begin{array}{l}\text { American Association of } \\
\text { Veterinary Parasitologists }\end{array}$ & AAV & aavp.org & 8 & Shawnee, KS & USA & No & 1956 \\
\hline 16 & $\begin{array}{l}\text { American College of Preventive } \\
\text { Medicine }\end{array}$ & $\mathrm{ACP}$ & acpm.org & 8 & Washington, DC & USA & Yes & 1954 \\
\hline 17 & $\begin{array}{l}\text { American International Health } \\
\text { Alliance }\end{array}$ & AlH & aiha.com & 6 & Washington, DC & USA & Yes & 1992 \\
\hline 18 & American Jewish World Service & AJW & ajws.org & 6 & New York City, NY & USA & No & 1985 \\
\hline 19 & American Public Health Association & $\mathrm{APH}$ & apha.org & 8 & Washington, DC & USA & Yes & 1872 \\
\hline 20 & American Red Cross & ARC & redcross.org & 6 & Washington, DC & USA & No & 1881 \\
\hline 21 & American Refugee Committee & ARO & arcrelief.org & 6 & Minneapolis, MN & USA & No & 1979 \\
\hline 22 & American Society for Microbiology & ASM & asm.org & 8 & Washington, DC & USA & No & 1899 \\
\hline 23 & $\begin{array}{l}\text { American Society of Tropical } \\
\text { Medicine and Hygiene }\end{array}$ & AST & astmh.org & 6 & Deerfield, IL & USA & Yes & 1903 \\
\hline 24 & American Thoracic Society & ATS & thoracic.org & 8 & New York City, NY & USA & Yes & 1905 \\
\hline 25 & $\begin{array}{l}\text { amfAR (Foundation for AIDS } \\
\text { Research) }\end{array}$ & AMA & amfar.org & 6 & New York City, NY & USA & Yes & 1985 \\
\hline 26 & Anaerobe Society of the Americas & ASA & anaerobe.org & 8 & Los Angeles, CA & USA & Yes & 1992 \\
\hline 27 & $\begin{array}{l}\text { Asia Pacific Malaria Elimination } \\
\text { Network }\end{array}$ & APM & apmen.org & 4 & Herston & Australia & Yes & 2009 \\
\hline 28 & $\begin{array}{l}\text { Association of Public Health } \\
\text { Laboratories }\end{array}$ & $\mathrm{APL}$ & aphl.org & 6 & Silver Spring, MD & USA & Yes & 1951 \\
\hline 29 & $\begin{array}{l}\text { Australasian College of Tropical } \\
\text { Medicine }\end{array}$ & ACM & tropmed.org & 8 & Brisbane & Australia & Yes & 1991 \\
\hline 30 & $\begin{array}{l}\text { AVAC: Global Advocacy for HIV } \\
\text { Prevention }\end{array}$ & AVA & avac.org & 6 & New York City, NY & USA & Yes & 1995 \\
\hline 31 & AVERT & AVE & avert.org & 6 & Horsham & UK & Yes & 1986 \\
\hline 32 & Bill \& Melinda Gates Foundation & BMG & gatesfoundation.org & 5 & Seattle, WA & USA & No & 2000 \\
\hline 33 & BIO Ventures for Global Health & BVG & bvgh.org & 6 & Seattle, WA & USA & Yes & 2004 \\
\hline 34 & Campaign for Tobacco-Free Kids & CTF & global.tobaccofreekids.org & 6 & Washington, DC & USA & Yes & 1995 \\
\hline 35 & CARE International & CAl & care-international.org & 6 & Geneva & Switzerland & No & 1945 \\
\hline
\end{tabular}


Table 2 List of global health actors included in the systematic review (Continued)

\begin{tabular}{|c|c|c|c|c|c|c|c|c|}
\hline \multicolumn{2}{|c|}{ Actor } & \multirow{2}{*}{$\begin{array}{l}\text { Code } \\
\text { CRI }\end{array}$} & \multirow{2}{*}{$\begin{array}{l}\text { URL } \\
\text { caritas.org }\end{array}$} & \multirow{2}{*}{$\begin{array}{l}\text { Type } \\
6\end{array}$} & \multicolumn{2}{|c|}{ Headquarters Location } & \multirow{2}{*}{$\begin{array}{l}\text { Health as } \\
\text { primary } \\
\text { intent? } \\
\text { No }\end{array}$} & \multirow{2}{*}{$\begin{array}{l}\text { Year of } \\
\text { Inceptior }\end{array}$} \\
\hline 36 & Caritas International & & & & Vatican City & $\begin{array}{l}\text { Vatican City } \\
\text { State }\end{array}$ & & \\
\hline 37 & Catholic Medical Mission Board & CMM & cmmb.org & 6 & New York City, NY & USA & Yes & 1912 \\
\hline 38 & Catholics for Choice & CFC & catholicsforchoice.org & 6 & Washington, DC & USA & No & 1973 \\
\hline 39 & CDC Foundation & CDC & cdcfoundation.org & 6 & Atlanta, GA & USA & Yes & 1995 \\
\hline 40 & Center for Global Development & CGD & cgdev.org & 6 & Washington, DC & USA & No & 2001 \\
\hline 41 & Center for Health and Gender Equity & $\mathrm{CHG}$ & genderhealth.org & 6 & Washington, DC & USA & No & 1994 \\
\hline 42 & $\begin{array}{l}\text { Center for International Environmental } \\
\text { Law }\end{array}$ & $\mathrm{CIE}$ & ciel.org & 6 & Washington, DC & USA & No & 1989 \\
\hline 43 & Center for Reproductive Rights & CRR & reproductiverights.org & 6 & New York City, NY & USA & No & 1992 \\
\hline 44 & $\begin{array}{l}\text { Centers for Disease Control and } \\
\text { Prevention }\end{array}$ & CDP & cdc.gov & 1 & Atlanta, GA & USA & Yes & 1946 \\
\hline 45 & Chemonics International & $\mathrm{CHI}$ & chemonics.com & 7 & Washington, DC & USA & No & 1975 \\
\hline 46 & $\begin{array}{l}\text { Christian Connections for } \\
\text { International Health }\end{array}$ & $\mathrm{CCl}$ & ccih.org & 6 & McLean, VA & USA & Yes & 1987 \\
\hline 47 & CONRAD & CON & conrad.org & 6 & Arlington, VA & USA & Yes & 1986 \\
\hline 48 & $\begin{array}{l}\text { Consultative Group on Early } \\
\text { Childhood Care and Development }\end{array}$ & CGE & ecdgroup.com & 6 & Toronto & Canada & No & 1984 \\
\hline 49 & CORE Group & COG & coregroup.org & 6 & Washington, DC & USA & Yes & 1997 \\
\hline 50 & Countdown to 2015 & СОТ & countdown2015mnch.org & 6 & Geneva & Switzerland & Yes & 2005 \\
\hline 51 & Direct Relief & $\mathrm{DIR}$ & directrelief.org & 6 & Santa Barbara, CA & USA & Yes & 1948 \\
\hline 52 & Doctors for Global Health & DGH & dghonline.org & 6 & Decatur, GA & USA & No & 1995 \\
\hline 53 & $\begin{array}{l}\text { Elizabeth Glaser Pediatric AIDS } \\
\text { Foundation }\end{array}$ & EGP & pedaids.org & 6 & Washington, DC & USA & Yes & 1988 \\
\hline 54 & Elton John AIDS Foundation & EJA & ejaf.org & 6 & London & UK & Yes & 1992 \\
\hline 55 & EngenderHealth & $\mathrm{ENH}$ & engenderhealth.org & 6 & New York City, NY & USA & Yes & 1943 \\
\hline 56 & Episcopal Relief \& Development & ERD & episcopalrelief.org & 6 & New York City, NY & USA & No & 1940 \\
\hline 57 & $\begin{array}{l}\text { European \& Developing Countries } \\
\text { Clinical Trials Partnership }\end{array}$ & EDC & edctp.org & 2 & The Hague & Netherlands & Yes & 2003 \\
\hline 58 & European AIDS Treatment Group & EAT & eatg.org & 6 & Brussels & Belgium & Yes & 1992 \\
\hline 59 & European Food Information Council & $\mathrm{EFI}$ & eufic.org & 6 & Brussels & Belgium & No & 1995 \\
\hline 60 & $\begin{array}{l}\text { European Generic Medicines } \\
\text { Association }\end{array}$ & EGM & egagenerics.com & 7 & Brussels & Belgium & No & 1993 \\
\hline 61 & $\begin{array}{l}\text { European Medical Students' } \\
\text { Association }\end{array}$ & EMS & emsa-europe.org & 6 & Brussels & Belgium & No & 1991 \\
\hline 62 & $\begin{array}{l}\text { European NGOs for Sexual and } \\
\text { Reproductive Health and Rights, } \\
\text { Population and Development }\end{array}$ & ENS & eurongos.org & 6 & Brussels & Belgium & Yes & 1996 \\
\hline 63 & $\begin{array}{l}\text { European Observatory on Health } \\
\text { Systems and Policies }\end{array}$ & $\mathrm{EOH}$ & $\begin{array}{l}\text { euro.who.int/en/about-us/ } \\
\text { partners/observatory }\end{array}$ & 4 & Brussels & Belgium & Yes & 1998 \\
\hline 64 & European Respiratory Society & ERS & ersnet.org & 8 & Lausanne & Switzerland & Yes & 1990 \\
\hline 65 & European Vaccine Initiative & EVI & euvaccine.eu & 6 & Heidelberg & Germany & Yes & 1998 \\
\hline 66 & Family Care International & $\mathrm{FCl}$ & familycareintl.org & 6 & New York City, NY & USA & Yes & 1986 \\
\hline 67 & $\begin{array}{l}\text { Federation of American Societies } \\
\text { for Experimental Biology }\end{array}$ & FAS & faseb.org & 8 & Bethesda, $\mathrm{OH}$ & USA & Yes & 1912 \\
\hline 68 & Feed the Future & FTF & feedthefuture.gov & 1 & Washington, DC & USA & No & 2010 \\
\hline 69 & $\begin{array}{l}\text { FHI } 360 \text { (formerly Family Health } \\
\text { International) }\end{array}$ & $\mathrm{FHI}$ & fhi360.org & 6 & Durham, NC & USA & No & 2011 \\
\hline
\end{tabular}


Table 2 List of global health actors included in the systematic review (Continued)

\begin{tabular}{|c|c|c|c|c|c|c|c|c|}
\hline \multicolumn{2}{|c|}{ Actor } & \multirow{2}{*}{$\begin{array}{l}\text { Code } \\
\text { FIF }\end{array}$} & \multirow{2}{*}{$\begin{array}{l}\text { URL } \\
\text { firelightfoundation.org }\end{array}$} & \multirow{2}{*}{$\begin{array}{l}\text { Type } \\
6\end{array}$} & \multicolumn{2}{|c|}{ Headquarters Location } & \multirow{2}{*}{$\begin{array}{l}\text { Health as } \\
\text { primary } \\
\text { intent? } \\
\text { No }\end{array}$} & \multirow{2}{*}{$\begin{array}{l}\text { Year of } \\
\text { Inception } \\
2000\end{array}$} \\
\hline 70 & Firelight Foundation & & & & Santa Cruz, CA & USA & & \\
\hline 71 & Fistula Foundation & FSF & fistulafoundation.org & 6 & San Jose, CA & USA & Yes & 2000 \\
\hline 72 & $\begin{array}{l}\text { Food and Agriculture Organization } \\
\text { of the United Nations }\end{array}$ & $\mathrm{FAO}$ & fao.org & 2 & Rome & Italy & No & 1945 \\
\hline 73 & $\begin{array}{l}\text { Foundation for Innovative New } \\
\text { Diagnostics }\end{array}$ & FIN & finddiagnostics.org & 6 & Geneva & Switzerland & Yes & 2003 \\
\hline 74 & $\begin{array}{l}\text { Foundation for International Medical } \\
\text { Relief of Children }\end{array}$ & FIM & fimrc.org & 6 & Philadelphia, PA & USA & Yes & 2002 \\
\hline 75 & $\begin{array}{l}\text { Framework Convention Alliance for } \\
\text { Tobacco Control }\end{array}$ & FCA & fctc.org & 6 & Geneva & Switzerland & No & 1999 \\
\hline 76 & Futures Group & FUG & futuresgroup.com & 7 & Washington, DC & USA & Yes & 1971 \\
\hline 77 & Gavi, the Vaccine Alliance & GAA & gavialliance.org & 4 & Geneva & Switzerland & Yes & 1999 \\
\hline 78 & GBCHealth & $\mathrm{GBH}$ & gbchealth.org & 6 & New York City, NY & USA & Yes & 2001 \\
\hline 79 & Global Advisors Smokefree Policy & GAS & njgasp.org & 6 & Summit, NJ & USA & No & 1974 \\
\hline 80 & $\begin{array}{l}\text { Global Alliance for TB Drug } \\
\text { Development }\end{array}$ & GAT & tballiance.org & 4 & New York City, NY & USA & Yes & 2000 \\
\hline 81 & $\begin{array}{l}\text { Global Coalition Against Child } \\
\text { Pneumonia }\end{array}$ & GCA & worldpneumoniaday.org & 4 & Baltimore, MD & USA & Yes & 2009 \\
\hline 82 & Global Communities & GLC & globalcommunities.org & 6 & Silver Spring, MD & USA & No & 1952 \\
\hline 83 & Global Health Corps & GHC & ghcorps.org & 6 & New York City, NY & USA & No & 2008 \\
\hline 84 & Global Health Council & $\mathrm{GHO}$ & globalhealth.org & 6 & Washington, DC & USA & Yes & 1972 \\
\hline 85 & Global Health Workforce Alliance & GHW & who.int/workforcealliance & 4 & Geneva & Switzerland & Yes & 2006 \\
\hline 86 & Global HIV Vaccine Enterprise & GHV & vaccineenterprise.org & 6 & New York City, NY & USA & Yes & 2004 \\
\hline 87 & Global Hope Network International & GHN & globalhopenetwork.org & 6 & Geneva & Switzerland & No & 1999 \\
\hline 88 & $\begin{array}{l}\text { Global Network of People Living } \\
\text { with HIV }\end{array}$ & GNP & gnpplus.net & 6 & Amsterdam & Netherlands & No & 1986 \\
\hline 89 & Guttmacher Institute & GUI & guttmacher.org & 6 & New York City, NY & USA & Yes & 1968 \\
\hline 90 & Health Action International & $\mathrm{HAl}$ & haiweb.org & 6 & Geneva & Switzerland & Yes & 1981 \\
\hline 91 & Health Skepticism Inc & HIS & healthyskepticism.org & 6 & Port Willunga & Australia & Yes & 1983 \\
\hline 92 & Health Volunteers Overseas & HVO & hvousa.org & 6 & Washington, DC & USA & Yes & 1986 \\
\hline 93 & HealthCare Volunteer & $\mathrm{HCV}$ & healthcarevolunteer.com & 6 & Los Altos, CA & USA & Yes & 2005 \\
\hline 94 & HealthRight International & $\mathrm{HRI}$ & healthright.org & 6 & New York City, NY & USA & Yes & 1990 \\
\hline 95 & Hellen Keller International & $\mathrm{HKI}$ & hki.org & 6 & New York City, NY & USA & Yes & 1915 \\
\hline 96 & Higher Education for Development & HED & hedprogram.org & 6 & Washington, DC & USA & No & 1918 \\
\hline 97 & $\begin{array}{l}\text { IBFAN (International Baby Food } \\
\text { Action Network) }\end{array}$ & IBF & ibfan.org & 6 & Geneva & Switzerland & Yes & 1979 \\
\hline 98 & Ibis Reproductive Health & $\mathrm{IRH}$ & ibisreproductivehealth.org & 6 & Cambridge, MA & USA & No & 2002 \\
\hline 99 & $\begin{array}{l}\text { ICASCO (International Council of } \\
\text { AIDS Service Organizations) }\end{array}$ & ICA & icaso.org & 6 & Toronto & Canada & Yes & 1991 \\
\hline 100 & Infectious Disease Research Institute & IDR & idri.org & 6 & Washington, DC & USA & Yes & 1993 \\
\hline 101 & Institute of Food Technologists & IFT & ift.org & 6 & Chicago, IL & USA & No & 1939 \\
\hline 102 & International AIDS Society & IAS & iasociety.org & 8 & Geneva & Switzerland & Yes & 1988 \\
\hline 103 & International AIDS Vaccine Initiative & IAV & iavi.org & 6 & New York City, NY & USA & Yes & 1996 \\
\hline 104 & $\begin{array}{l}\text { International Association for Food } \\
\text { Protection }\end{array}$ & IAF & foodprotection.org & 6 & Des Moines, IA & USA & No & 1911 \\
\hline 105 & International Association of National & IAN & ianphi.org & 2 & Atlanta, GA & USA & Yes & 2006 \\
\hline
\end{tabular}


Table $\mathbf{2}$ List of global health actors included in the systematic review (Continued)

\begin{tabular}{|c|c|c|c|c|c|c|c|c|}
\hline \multicolumn{2}{|c|}{ Actor } & \multirow{2}{*}{$\begin{array}{l}\text { Code } \\
\text { IAP }\end{array}$} & \multirow{2}{*}{$\begin{array}{l}\text { URL } \\
\text { iapac.org }\end{array}$} & \multirow{2}{*}{$\begin{array}{l}\text { Type } \\
8\end{array}$} & \multicolumn{2}{|c|}{ Headquarters Location } & \multirow{2}{*}{$\begin{array}{l}\text { Health as } \\
\text { primary } \\
\text { intent? } \\
\text { Yes }\end{array}$} & \multirow{2}{*}{$\begin{array}{l}\text { Year of } \\
\text { Inception }\end{array}$} \\
\hline 106 & $\begin{array}{l}\text { International Association of Providers } \\
\text { of AIDS Care }\end{array}$ & & & & Chicago, IL & USA & & \\
\hline 107 & $\begin{array}{l}\text { International Center for Research on } \\
\text { Women }\end{array}$ & $I C R$ & icrw.org & 6 & Washington, DC & USA & No & 1976 \\
\hline 108 & $\begin{array}{l}\text { International Consortium for } \\
\text { Emergency Contraception }\end{array}$ & ICE & cecinfo.org & 6 & New York City, NY & USA & Yes & 1996 \\
\hline 109 & $\begin{array}{l}\text { UNITAID (International Drug } \\
\text { Purchase Facility) }\end{array}$ & IDP & unitaid.eu & 4 & Geneva & Switzerland & Yes & 2006 \\
\hline 110 & $\begin{array}{l}\text { International Epidemiological } \\
\text { Association }\end{array}$ & IEA & ieaweb.org & 8 & Raleigh, NC & USA & No & 1954 \\
\hline 111 & $\begin{array}{l}\text { International Federation of Medical } \\
\text { Students' Associations }\end{array}$ & IFM & ifmsa.org & 6 & Amsterdam & Netherlands & No & 1951 \\
\hline 112 & $\begin{array}{l}\text { International Finance Facility for } \\
\text { Immunisation }\end{array}$ & IFF & iffim.org & 6 & London & UK & Yes & 2006 \\
\hline 113 & $\begin{array}{l}\text { International Food Policy Research } \\
\text { Institute }\end{array}$ & IFP & ifpri.org & 6 & Washington, DC & USA & No & 1975 \\
\hline 114 & $\begin{array}{l}\text { International Fund for Agricultural } \\
\text { Development }\end{array}$ & IFA & ifad.org & 2 & Rome & Italy & No & 1977 \\
\hline 115 & International Health Partnership & $\mathrm{HP}$ & internationalhealthpartnership.net & 4 & Washington, DC & USA & Yes & 2007 \\
\hline 116 & International HIV/AIDS Alliance & $\mathrm{HA}$ & aidsalliance.org & 6 & Hove & UK & Yes & 1993 \\
\hline 117 & International Life Sciences Institute & ILS & ilsi.org & 6 & Washington, DC & USA & No & 1978 \\
\hline 118 & $\begin{array}{l}\text { International Network for Rational } \\
\text { Use of Drugs }\end{array}$ & INR & inrud.org & 4 & Arlington, VA & USA & No & 1989 \\
\hline 119 & $\begin{array}{l}\text { International Partnership for } \\
\text { Microbicides }\end{array}$ & IPM & ipmglobal.org & 4 & Silver Spring, MD & USA & Yes & 2002 \\
\hline 120 & $\begin{array}{l}\text { International Pharmaceutical } \\
\text { Students' Federation }\end{array}$ & IPS & ipsf.org & 6 & The Hague & Netherlands & Yes & 1949 \\
\hline 121 & $\begin{array}{l}\text { International Planned Parenthood } \\
\text { Federation }\end{array}$ & $\mathrm{IPP}$ & ippf.org & 6 & London & UK & No & 1952 \\
\hline 122 & International Relief \& Development & IRD & ird.org & 6 & Arlington, VA & USA & No & 1998 \\
\hline 123 & $\begin{array}{l}\text { International Society for Infectious } \\
\text { Diseases }\end{array}$ & $|S|$ & isid.org & 6 & Brookline, MA & USA & Yes & 1986 \\
\hline 124 & International Society of Drug Bulletins & ISD & isdbweb.org & 6 & London & UK & No & 1986 \\
\hline 125 & $\begin{array}{l}\text { International Union Against } \\
\text { Tuberculosis and Lung Disease }\end{array}$ & IUA & theunion.org & 4 & Paris & France & Yes & 1920 \\
\hline 126 & $\begin{array}{l}\text { International Union of Food Science } \\
\text { and Technology }\end{array}$ & IUF & iufost.org & 6 & Oakville & Canada & No & 1970 \\
\hline 127 & $\begin{array}{l}\text { International Union of Nutritional } \\
\text { Sciences }\end{array}$ & IUN & iuns.org & 6 & Vienna & Austria & No & 1948 \\
\hline 128 & International Vaccine Institute & $\mid \mathrm{VI}$ & ivi.int & 6 & Seoul & $\begin{array}{l}\text { Republic of } \\
\text { Korea }\end{array}$ & Yes & 1996 \\
\hline 129 & IntraHealth International & $\| \mathrm{H}$ & intrahealth.org & 6 & Chapel Hill, NC & USA & Yes & 1979 \\
\hline 130 & $\begin{array}{l}\text { Ipas (formerly International } \\
\text { Pregnancy Advisory Services) }\end{array}$ & $\mathrm{IPA}$ & ipas.org & 6 & Chapel Hill, NC & USA & Yes & 1973 \\
\hline 131 & Jhpiego & JHP & jhpiego.org & 6 & Baltimore, MD & USA & Yes & 1974 \\
\hline 132 & John Snow, Inc. & JSI & jsi.com & 7 & Boston, MA & USA & Yes & 1978 \\
\hline 133 & $\begin{array}{l}\text { Johns Hopkins Bloomberg School } \\
\text { of Public Health }\end{array}$ & $J H B$ & jhsph.edu & 9 & Baltimore, MD & USA & Yes & 1916 \\
\hline 134 & Joint United Nations Programme & $J U N$ & unaids.org & 2 & Geneva & Switzerland & Yes & 1996 \\
\hline
\end{tabular}


Table $\mathbf{2}$ List of global health actors included in the systematic review (Continued)

\begin{tabular}{|c|c|c|c|c|c|c|c|c|}
\hline \multicolumn{2}{|c|}{ Actor } & \multirow{2}{*}{$\begin{array}{l}\text { Code } \\
\text { LSH }\end{array}$} & \multirow{2}{*}{$\begin{array}{l}\text { URL } \\
\text { www.Ishtm.ac.uk }\end{array}$} & \multirow{2}{*}{$\begin{array}{l}\text { Type } \\
9\end{array}$} & \multicolumn{2}{|c|}{ Headquarters Location } & \multirow{2}{*}{$\begin{array}{l}\text { Health as } \\
\text { primary } \\
\text { intent? } \\
\text { Yes }\end{array}$} & \multirow{2}{*}{$\begin{array}{l}\text { Year of } \\
\text { Inception }\end{array}$} \\
\hline 135 & $\begin{array}{l}\text { London School of Hygiene and } \\
\text { Tropical Medicine }\end{array}$ & & & & London & UK & & \\
\hline 136 & Malaria Foundation International & $\mathrm{MFI}$ & malaria.org & 6 & $\begin{array}{l}\text { Stone Mountain, } \\
\text { GA }\end{array}$ & USA & Yes & 1992 \\
\hline 137 & Malaria No More & MNM & malarianomore.org & 6 & New York City, NY & USA & Yes & 2006 \\
\hline 138 & Management Systems International & MSN & msiworldwide.com & 7 & Washington, DC & USA & No & 1981 \\
\hline 139 & Médecins Sans Frontières & MSF & msf.org & 6 & Geneva & Switzerland & Yes & 1971 \\
\hline 140 & Medicines for Malaria Venture & MMV & mmv.org & 4 & Geneva & Switzerland & Yes & 1999 \\
\hline 141 & MediSend International & MSI & medisend.org & 6 & Dallas, TX & USA & Yes & 1999 \\
\hline 142 & Mercy Corps & MEC & mercycorps.org & 6 & Portland, OR & USA & No & 1979 \\
\hline 143 & Millennium Challenge Corporation & MCC & mcc.gov & 1 & Washington, DC & USA & No & 2004 \\
\hline 144 & National Institutes of Health & $\mathrm{NIH}$ & nih.gov & 1 & Bethesda, $\mathrm{OH}$ & USA & Yes & 1887 \\
\hline 145 & Operation Rainbow & OPR & operationrainbow.org & 6 & Oakland, CA & USA & Yes & 1978 \\
\hline 146 & Operation Smile & OPS & operationsmile.org & 6 & Virginia Beach, VA & USA & Yes & 1982 \\
\hline 147 & Operation USA & OPU & opusa.org & 6 & Los Angeles, CA & USA & No & 1979 \\
\hline 148 & Oxfam International & OXI & oxfam.org & 6 & Washington, DC & USA & No & 1995 \\
\hline 149 & $\begin{array}{l}\text { Pan American Society for Clinical } \\
\text { Virology }\end{array}$ & PAS & pascv.org & 8 & Raleigh, NC & USA & Yes & 1977 \\
\hline 150 & Pangaea Global AIDS Foundation & PGA & pangaeaglobal.org & 6 & Oakland, CA & USA & Yes & 2001 \\
\hline 151 & Partners in Health & $\mathrm{PIH}$ & pih.org & 6 & Boston, MA & USA & Yes & 1987 \\
\hline 152 & $\begin{array}{l}\text { Partnership for Maternal, Newborn } \\
\text { and Child Health }\end{array}$ & PMN & who.int/pmnch & 4 & Geneva & Switzerland & Yes & 2005 \\
\hline 153 & PATH & PAT & path.org & 6 & Seattle, WA & USA & Yes & 1977 \\
\hline 154 & Pathfinder International & PAl & pathfinder.org & 6 & Watertown, MN & USA & Yes & 1957 \\
\hline 155 & Pediatric Infectious Diseases Society & PID & pids.org & 8 & Arlington, VA & USA & Yes & 1984 \\
\hline 156 & Plan International & PLI & plan-international.org & 6 & Woking & UK & No & 1937 \\
\hline 157 & Population Action International & PAN & populationaction.org & 6 & Washington, DC & USA & No & 1965 \\
\hline 158 & Population Council & POC & popcouncil.org & 6 & New York City, NY & USA & No & 1952 \\
\hline 159 & Population Media Center & PMC & populationmedia.org & 6 & Shelburne, MA & USA & Yes & 1998 \\
\hline 160 & Population Reference Bureau & PRB & prb.org & 6 & Washington, DC & USA & No & 1929 \\
\hline 161 & Population Services International & PSI & psi.org & 6 & Washington, DC & USA & Yes & 1970 \\
\hline 162 & Project HOPE & $\mathrm{PRH}$ & projecthope.org & 6 & Millwood, VA & USA & Yes & 1958 \\
\hline 163 & Public Health Institute & $\mathrm{PHI}$ & phi.org & 6 & Oakland, CA & USA & Yes & 1964 \\
\hline 164 & RAND Corporation & RAC & rand.org & 6 & Santa Monica, CA & USA & No & 1948 \\
\hline 165 & Refugees International & REI & refintl.org & 6 & Washington, DC & USA & No & 1979 \\
\hline 166 & $\begin{array}{l}\text { Reproductive Health Response in } \\
\text { Crises Consortium }\end{array}$ & $\mathrm{RHR}$ & rhrc.org & 6 & Minneapolis, MN & USA & Yes & 1995 \\
\hline 167 & $\begin{array}{l}\text { Reproductive Health Supplies } \\
\text { Coalition }\end{array}$ & RHS & rhsupplies.org & 4 & Brussels & Belgium & Yes & 2004 \\
\hline 168 & $\begin{array}{l}\text { Research Triangle Institute } \\
\text { International }\end{array}$ & RTI & rti.org & 6 & $\begin{array}{l}\text { Durham } \\
\text { (Research Triangle } \\
\text { Park), NC }\end{array}$ & USA & No & 1958 \\
\hline 169 & Roll Back Malaria Partnership & RBM & rollbackmalaria.org & 4 & Geneva & Switzerland & Yes & 1998 \\
\hline 170 & Sabin Vaccine Institute & SVI & sabin.org & 6 & Washington, DC & USA & Yes & 1993 \\
\hline 171 & Save the Children International & $\mathrm{SCl}$ & savethechildren.net & 6 & London & UK & No & 1919 \\
\hline 172 & Society for Public Health Education & $\mathrm{SPH}$ & sophe.org & 8 & Washington, DC & USA & Yes & 1950 \\
\hline
\end{tabular}


Table $\mathbf{2}$ List of global health actors included in the systematic review (Continued)

\begin{tabular}{|c|c|c|c|c|c|c|c|c|}
\hline \multicolumn{2}{|c|}{ Actor } & \multirow{2}{*}{$\begin{array}{l}\text { Code } \\
\text { SRN }\end{array}$} & \multirow{2}{*}{$\begin{array}{l}\text { URL } \\
\text { srnt.org }\end{array}$} & \multirow{2}{*}{$\begin{array}{l}\text { Type } \\
6\end{array}$} & \multicolumn{2}{|c|}{ Headquarters Location } & \multirow{2}{*}{$\begin{array}{l}\text { Health as } \\
\text { primary } \\
\text { intent? } \\
\text { No }\end{array}$} & \multirow{2}{*}{$\begin{array}{l}\begin{array}{l}\text { Year of } \\
\text { Inception }\end{array} \\
1994\end{array}$} \\
\hline 173 & $\begin{array}{l}\text { Society for Research on Nicotine and } \\
\text { Tobacco }\end{array}$ & & & & Madison, Wl & USA & & \\
\hline 174 & Stephen Lewis Foundation & SLF & stephenlewisfoundation.org & 6 & Toronto & Canada & Yes & 2003 \\
\hline 175 & Stop TB Partnership & STP & stoptb.org & 4 & Geneva & Switzerland & Yes & 2001 \\
\hline 176 & $\begin{array}{l}\text { Swiss Tropical and Public Health } \\
\text { Institute }\end{array}$ & STH & swisstph.ch & 1 & Basel & Switzerland & Yes & 1943 \\
\hline 177 & Syrian Center for Tobacco Studies & $\mathrm{SCT}$ & scts-sy.org & 6 & Aleppo & $\begin{array}{l}\text { Syrian Arab } \\
\text { Republic }\end{array}$ & No & 2002 \\
\hline 178 & TB Alert & TBA & tbalert.org & 6 & Brighton & UK & Yes & 1998 \\
\hline 179 & The Earth Institute, Columbia University & TEl & earthinstitute.columbia.edu & 9 & New York City, NY & USA & No & 1995 \\
\hline 180 & $\begin{array}{l}\text { The Global Fund to Fight AIDS, } \\
\text { Tuberculosis and Malaria }\end{array}$ & TGF & theglobalfund.org & 4 & Geneva & Switzerland & Yes & 2002 \\
\hline 181 & The Water Project & TWP & thewaterproject.org & 6 & Concord, NH & USA & No & 2006 \\
\hline 182 & Tobacco Free Nurses & TFN & tobaccofreenurses.org & 6 & Los Angeles, CA & USA & No & 2003 \\
\hline 183 & Tostan & TOS & tostan.org & 6 & Dakar & Senegal & No & 1974 \\
\hline 184 & Treatment Action Group & TAG & treatmentactiongroup.org & 6 & New York City, NY & USA & Yes & 1992 \\
\hline 185 & TuBerculosis Vaccine Initiative & $\mathrm{TVI}$ & tbvi.eu & 6 & Lelystad & Netherlands & Yes & 2008 \\
\hline 186 & Unite for Sight & UFS & uniteforsight.org & 6 & New Haven, CT & USA & Yes & 2000 \\
\hline 187 & United Nations Children's Fund & UNC & unicef.org & 2 & New York City, NY & USA & No & 1946 \\
\hline 188 & $\begin{array}{l}\text { United Nations Development } \\
\text { Programme }\end{array}$ & UND & undp.org & 2 & New York City, NY & USA & No & 1966 \\
\hline 189 & United Nations Foundation & UNF & unfoundation.org & 6 & Washington, DC & USA & No & 1998 \\
\hline 190 & United Nations Population Fund & UNP & unfpa.org & 2 & Geneva & Switzerland & No & 1969 \\
\hline 191 & $\begin{array}{l}\text { United States Agency for } \\
\text { International Development }\end{array}$ & USA & usaid.gov & 1 & Washington, DC & USA & No & 1961 \\
\hline 192 & $\begin{array}{l}\text { United States Department of Health } \\
\text { \& Human Services, Office of Global } \\
\text { Affairs }\end{array}$ & USD & globalhealth.gov & 1 & Washington, DC & USA & Yes & 2002 \\
\hline 193 & University of California, San Francisco & UCS & www.ucsf.edu & 9 & San Francisco, CA & USA & Yes & 1864 \\
\hline 194 & VSO (Voluntary Service Overseas) & VSO & vso.org.uk & 6 & $\begin{array}{l}\text { Kingston upon } \\
\text { Thames }\end{array}$ & UK & No & 1958 \\
\hline 195 & Women Deliver & WOD & womendeliver.org & 6 & New York City, NY & USA & Yes & 2007 \\
\hline 196 & World AIDS Campaign & WAC & worldaidscampaign.org & 6 & Cape Town & South Africa & Yes & 1997 \\
\hline 197 & World Bank & WOB & worldbank.org & 3 & Washington, DC & USA & No & 1944 \\
\hline 198 & World Food Programme & WFP & wfp.org & 2 & Rome & Italy & No & 1961 \\
\hline 199 & World Health Organization & WHO & who.int & 2 & Geneva & Switzerland & Yes & 1948 \\
\hline 200 & World Lung Foundation & WLF & worldlungfoundation.org & 6 & New York City, NY & USA & Yes & 2004 \\
\hline 201 & World Vision International & WVI & wvi.org & 6 & Uxbridge & UK & No & 1950 \\
\hline 202 & Worldwatch Institute & WOI & worldwatch.org & 6 & Washington, DC & USA & No & 1974 \\
\hline 203 & Yale School of Public Health & YSP & publichealth.yale.edu & 9 & New Haven, CT & USA & Yes & 1946 \\
\hline
\end{tabular}

The numerical codes in the column labeled 'Type' represent the following actor types:

$1=$ National governments

$2=$ United Nations entities and intergovernmental organizations

$3=$ Multilateral development banks

$4=$ Public-private partnerships

$5=$ Philanthropic organizations

$6=$ Global civil society and non-governmental organizations

$7=$ Private industry

$8=$ Professional associations

$9=$ Academic institutions 
Table 3 Top ten global health actors by social network metrics

\begin{tabular}{|c|c|c|}
\hline Rank & Actor & Degree \\
\hline 1 & WHO & 48 \\
\hline 2 & $\mathrm{GHO}$ & 40 \\
\hline 3 & $\mathrm{FHI}$ & 39 \\
\hline 4 & TGF & 38 \\
\hline 5 & USA & 37 \\
\hline 6 & POC & 34 \\
\hline 7 & MSF & 31 \\
\hline 8 & PAN & 29 \\
\hline 9 & CDP & 23 \\
\hline 10 & UNC & 23 \\
\hline Rank & Actor & Closeness centrality \\
\hline 1 & WHO & 1.868020305 \\
\hline 2 & TGF & 2.208121827 \\
\hline 3 & $\mathrm{GHO}$ & 2.279187817 \\
\hline 4 & $\mathrm{FHI}$ & 2.319796954 \\
\hline 5 & USA & 2.324873096 \\
\hline 6 & MSF & 2.426395939 \\
\hline 7 & PAN & 2.456852792 \\
\hline 8 & UNC & 2.512690355 \\
\hline 9 & MFI & 2.532994924 \\
\hline 10 & CDP & 2.558375635 \\
\hline Rank & Actor & Betweenness centrality \\
\hline 1 & WHO & 2986.842403 \\
\hline 2 & $\mathrm{GHO}$ & 607.1230453 \\
\hline 3 & TGF & 557.3348557 \\
\hline 4 & CDP & 488.7137265 \\
\hline 5 & MSF & 485.1809524 \\
\hline 6 & USA & 394.0630366 \\
\hline 7 & UNC & 352.6553336 \\
\hline 8 & $\mathrm{FHI}$ & 297.9844801 \\
\hline 9 & STP & 259.9629191 \\
\hline 10 & POC & 234.107373 \\
\hline
\end{tabular}

academic institutions $(n=5)$, multilateral development banks $(n=1)$ and philanthropic institutions $(n=1)$ (see Fig. 2).

\section{Location}

International headquarters of the 203 global health actors were located in 16 countries and 73 cities (see Fig. 3). 98.5\% of headquarters were located in high-income countries. Two actors' headquarters were located in low- or lower-middle-income countries (i.e., Syrian Arab Republic and Senegal) and one in an upper-middle-income country (i.e., South Africa). The most common countries for global health actors to headquarter themselves were the U.S. $(n=135)$, Switzerland $(n=23)$, and the United Kingdom $(n=13)$, followed by Belgium $(n=7)$, The Netherlands $(n=6)$, and Canada $(n=4)$. The top three most common cities for headquarters were Washington, D.C. $(n=42)$, New York City $(n=28)$, and Geneva $(n=21)$.

\section{Year of inception}

Identified global health actors were created between 1864 and 2011, with the rate of inception of actors over time displayed in Fig. 4 and the inception of actors over time displayed as a network in Fig. 5.

\section{Primary intent}

$61.6 \%$ of global health actors $(n=125)$ listed improving health as the sole primary intent of their organization, compared to $38.4 \%$ of actors $(n=78)$ who listed improving health as one of multiple primary intents (see Fig. 6).

\section{Validation exercise}

The key global health system stakeholders who were consulted on the study's findings agreed that the definition developed for the global health system was analytically helpful. To their knowledge, the research was noted to be the first systematic attempt to map the global health system, and that having access to the basic list of actors was helpful. However, the participants agreed the resulting map of 203 actors did not comprehensively present the most important and influential actors in the global health system. The exercise revealed that senior leaders at major global health organizations value global representativeness, and that the results of future studies would be more helpful if their findings were generalizable at the global level.

\section{Discussion \\ Principal findings}

Our definition of the global health system was used effectively in a novel search methodology that took advantage of one of the world's most powerful Internet crawlers and related search algorithms (i.e., Google "related:URL" search). The methodology proved useful and efficient, systematically generating the largest network of global health actors to date. Notably, all identified actors were organizations. However, the network did include organizations founded by individuals, such as the Bill \& Melinda Gates Foundation, Elizabeth Glaser Pediatric AIDS Foundation, and Elton John AIDS Foundation. This reflects the tendency for websites to be created by organizations, whereas individuals tend to use social media or web pages on organizations' websites for their 

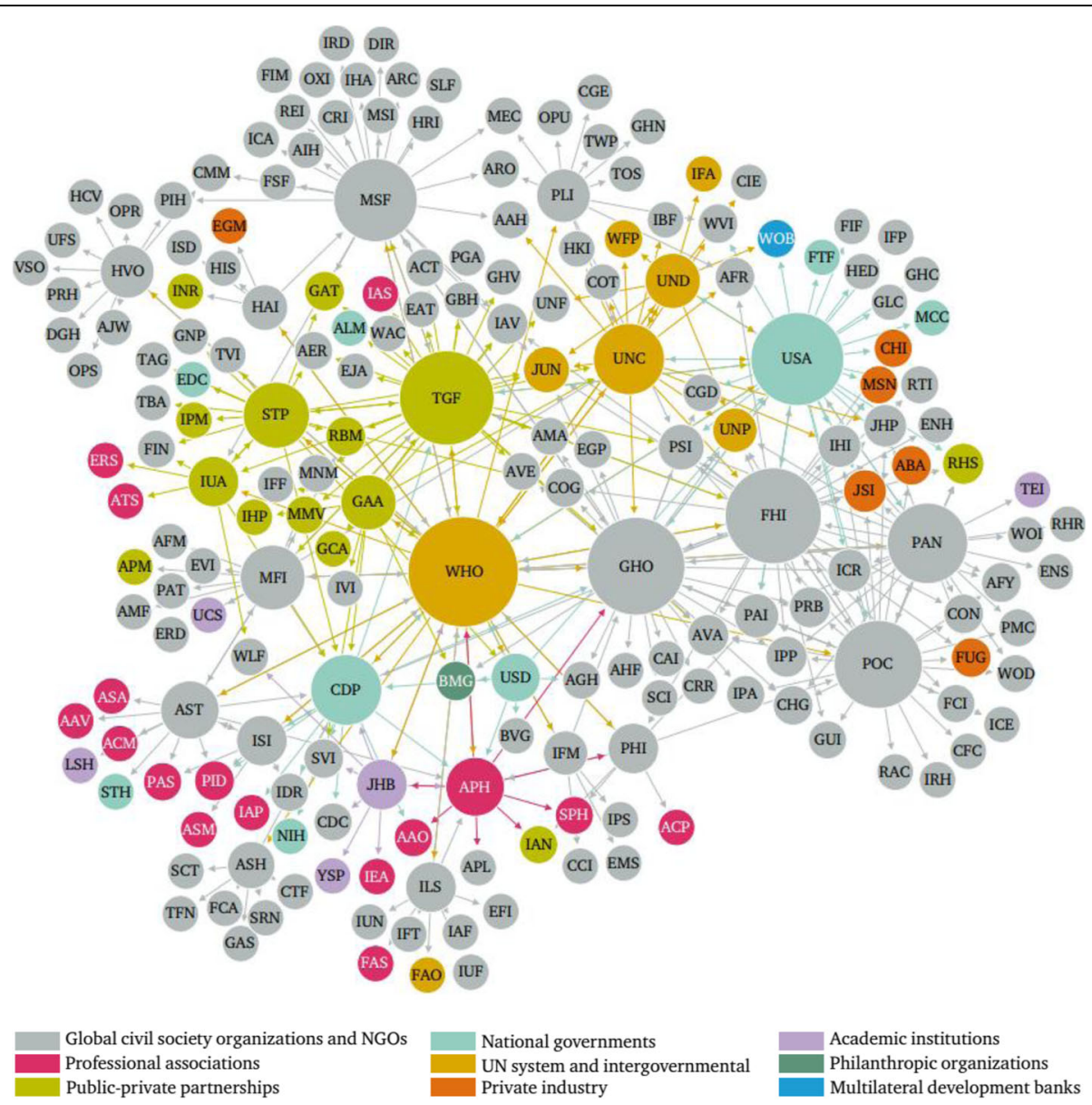

Fig. 2 Network mapping of global health actors by type. Node size is ranked by degree; node colour is partitioned by type of actor; and edges are coloured by source node

online presence (e.g., Facebook pages and Twitter accounts representing celebrities or university webpages featuring faculty members).

Basic analysis of the network structure reveals interesting findings about the online network of global health actors that may shed light on the offline global health system structure. The 26 global health actors identified in the search "related:www.who.int", which subsequent searches were based upon, are almost identical to the top 26 global health actors by degree centrality (after WHO, which ranks first). The only exception was the International Federation of Medical Students' Associations, which was identified in the first related search but had one too few connections to rank in the top 27 actors by degree. Interestingly, Population Services International and UNAIDS - two organizations that were not identified in the first related search and therefore whose placements in the network were not dictated by the search methodology - tied for the 27th ranking by degree. This indicates that the related search function found them to be highly related to many other websites.
They are placed in relatively central position in the online network in terms of activity. Offline, this may suggest Population Services International and UNAIDS are relatively well-connected and active players in the global health system.

Within the group of 26 actors identified through the first related search, there were eight organizations that consistently ranked in the top ten global health actors after WHO by degree, betweenness centrality, and closeness centrality: FHI360, Global Fund to Fight AIDS, Tuberculosis \& Malaria, Global Health Council, Médecins Sans Frontières, Stop TB Partnership, U.S. Agency for International Development, U.S. Centers for Disease Control and Prevention, and UNICEF. This result indicates the websites of these actors are central to the network: they are related to a relatively large number of other actors' websites, probably important in facilitating connections between websites, and allow for efficient connections to other actors in the system.

Through basic characterization of global health actors, various conclusions can be drawn. Categorization of 


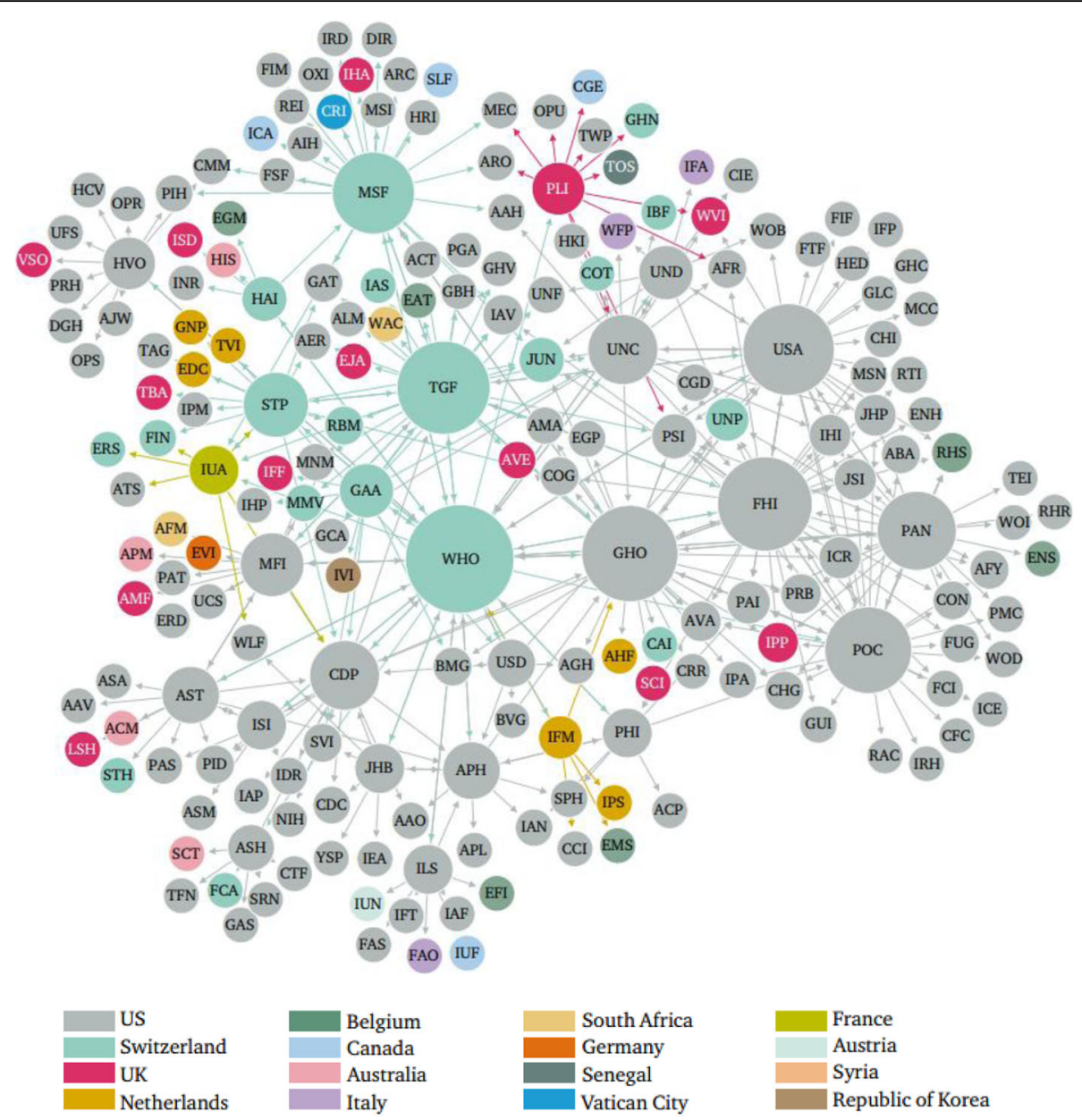

Fig. 3 Network mapping of global health actors by country location of international headquarters. Node size is ranked by degree, node colour is partitioned by headquarters location, and edges are coloured by source node

global health actors by type indicates the overwhelming presence of global civil society organizations and nongovernmental organizations in the online network of global health actors. The emergence of global health public-private partnerships is seen through an online presence that makes up just over $9 \%$ of identified global health actors [3]. Despite overwhelming influence of actors like the Bill \& Melinda Gates Foundation, [21] philanthropic organizations represented only $0.5 \%$ of identified global health actors. Visualization of the network by type of actor shows actors of the same type in distinct groupings, such as those of public-private partnerships, UN entities and intergovernmental organizations, and private industry respectively, suggesting that websites of the same type of global health actors are related to one another. Interestingly, the pre-chosen focal point of the global health system - the WHO - is directly connected to all actor types with the exception of philanthropic organizations and multilateral development banks, for which only one actor was identified in each category.
Geographical distribution of actors' international headquarters, spanning 73 cities across 16 countries, points to the global nature of the system. However, the overwhelming presence of actors' headquarters in high-income countries - more than half in the U.S. - clearly suggests an uneven distribution of actors' leadership globally. Significantly, no actor websites were located in the BRIC countries (i.e., Brazil, Russia, India and China). Several major centres of activity were identified, notably Washington, D.C., New York City, and Geneva. This reflects the location of various influential actors - global health and non-global health alike - around which global health actors have decided to co-locate. For example, the U.S. government and World Bank are headquartered in Washington, D.C., the UN is based in New York City, and WHO is in Geneva.

The inception of new global health actors over time indicates three distinct phases of rapid growth. The rate of inception of global health actors remained low until 1945, when a period of increased growth began, continuing until 1952. This growth coincides with the creation 


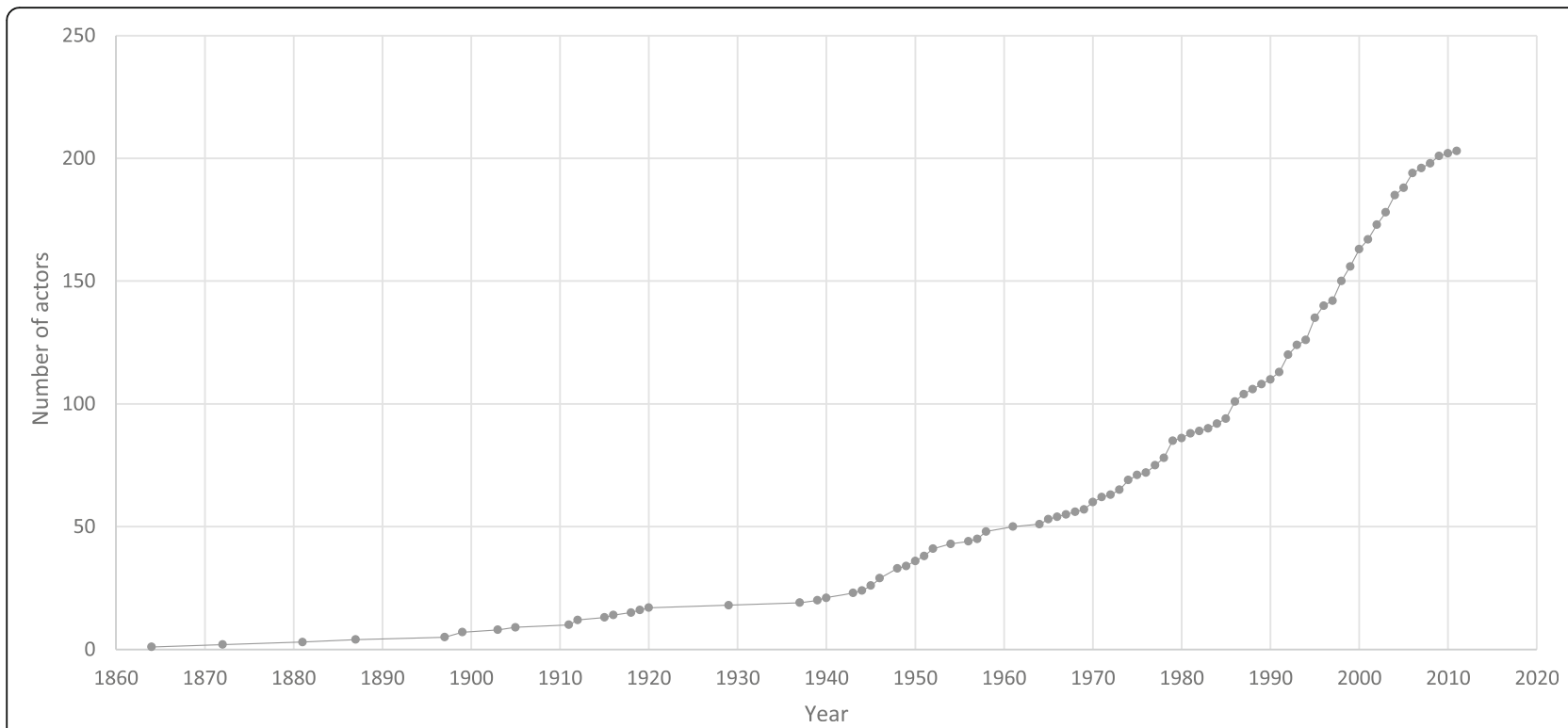

Fig. 4 Rate of inception of global health actors over time
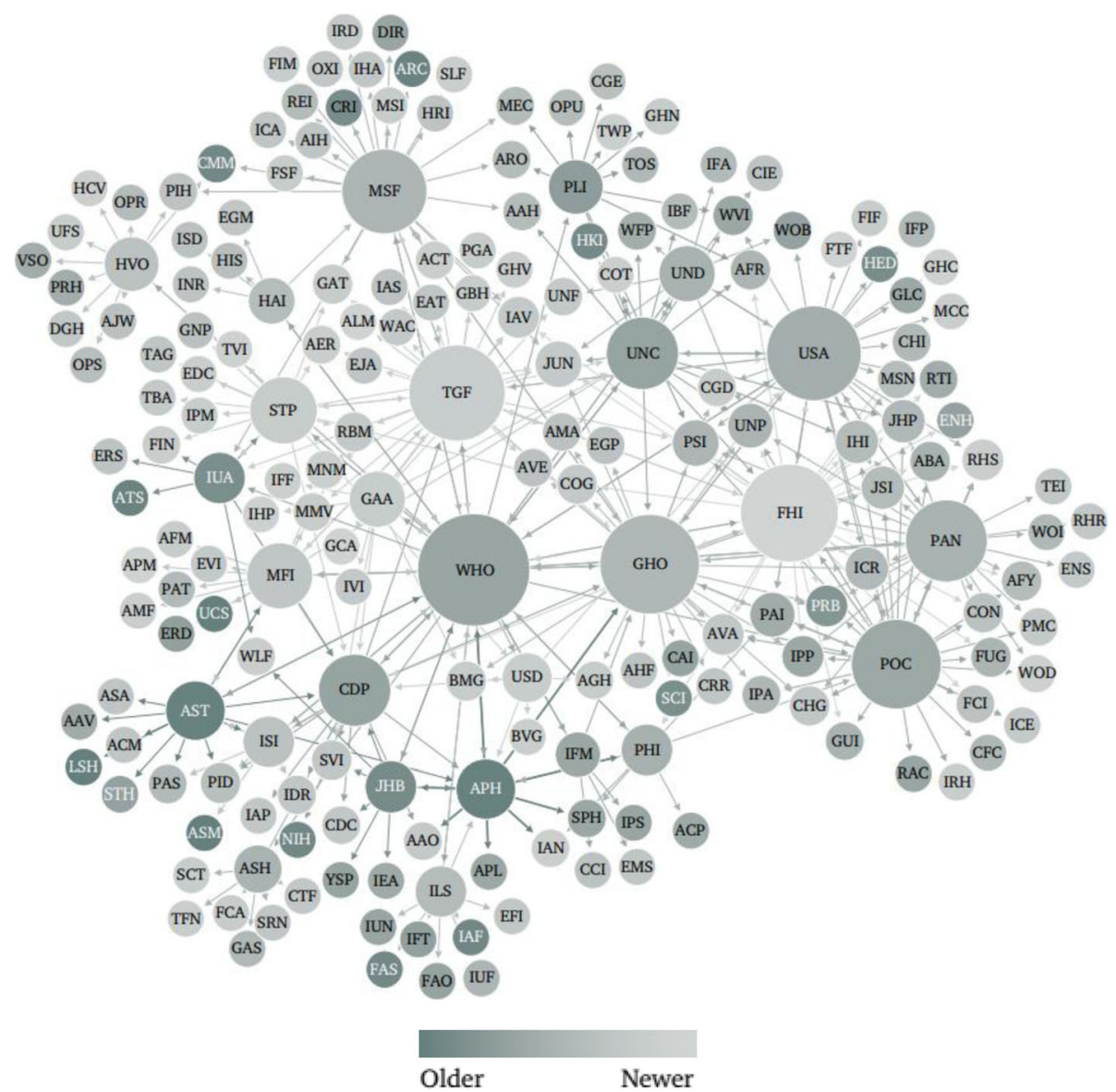

Fig. 5 Network mapping of global health actors by year of inception. Node size is ranked by degree; node colour is ranked by year of inception, where darker tones indicate an earlier year of inception and lighter ones indicate newer actors 


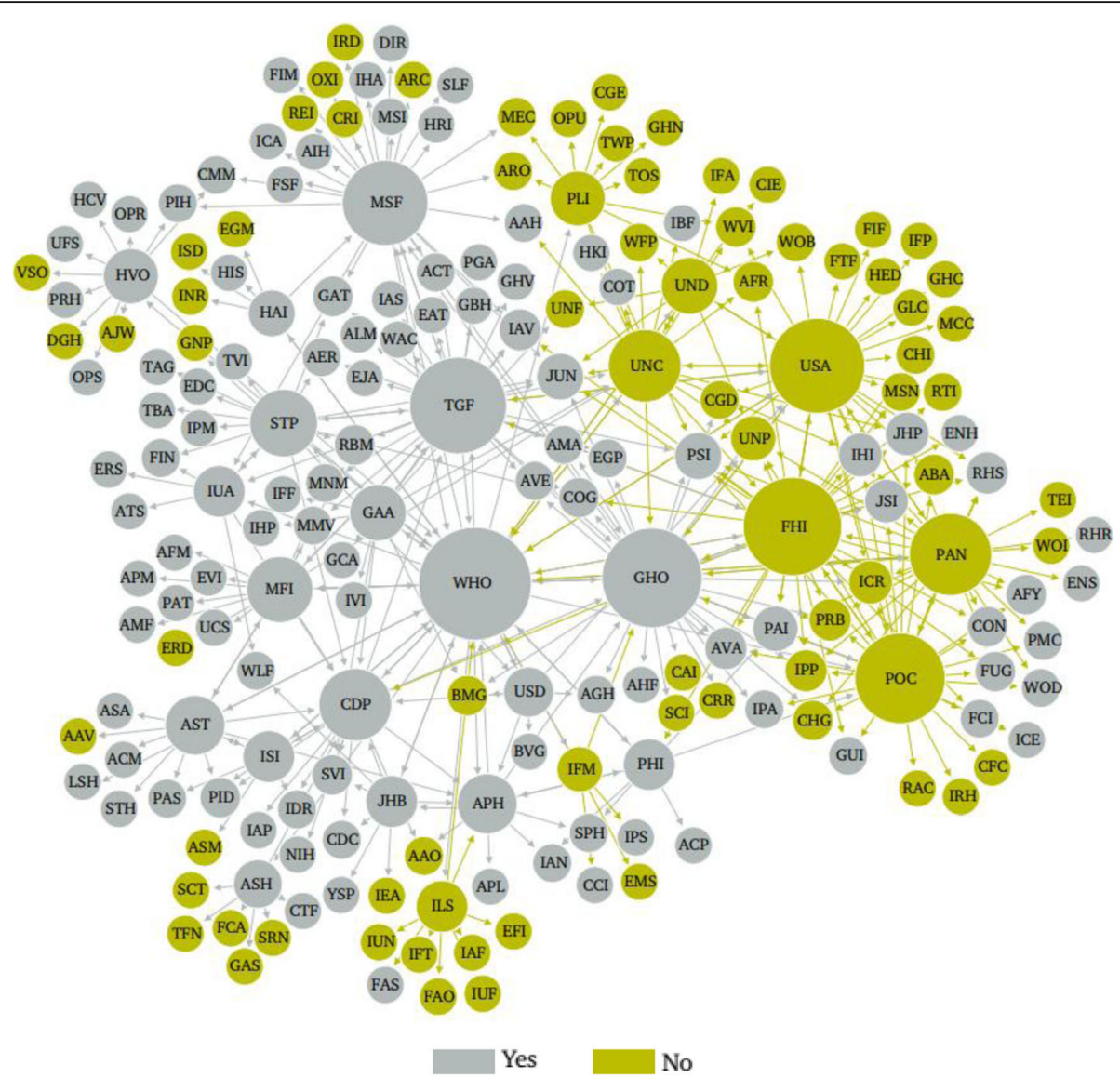

Fig. 6 Network mapping of global health actors by intent to improve health. Node size is ranked by degree; node colour is partitioned by whether or not improving health is the primary intent of the organization; and edges are coloured by source node

of the UN system in 1945 [22]. New global health actors may have been created as part of the nascent UN system itself, or in tandem with its development and also with that of other important multilateral organizations. A second phase of expansion began in 1970 and lasts through the decade, with a peak occurring in 1979. This expansion may reflect increased interest in international economic development at that time. For example, in 1973 the World Bank announced its pledge to increase financing for development by $40 \%$ over the following 5 years [23]. Last, an increase in actors is seen beginning in 1986 and continuing until 2006, coinciding with a quintupling of global health financing [24]. Each subsequent surge in the creation of new actors occurs at a higher rate than the previous one: an average of 2.00 actors were established per year from 1945 to 1952, 2.55 per year from 1970 to 1979 , and 4.91 per year from 1986 to 2006). This reflects an increase in the rate of growth of the global health system over time. The period of analysis ends with a lull in inception of new actors, due in part to the decade being incomplete, but also probably due to how emerging actors' websites may need time to gain relevance and popularity online and the 2008 global financial crisis.

Visualization of the network by year of inception shows newer global health actors in more central positions, and older organizations on the periphery of the network, indicating that the most influential actors in the global health system may not necessarily be the most established ones.

The proportion of actors whose primary intent is to improve health $(61.6 \%)$, versus those who describe improving health as one of their primary intents (38.4\%), illustrates the complex interaction between health and other global policy domains such as economic development and environmental protection. Network visualization shows some trends according to primary intent, such as in the high number of organizations for which improving health was not the primary intent that are connected to the U.S. Agency for International Development and the International Life Sciences Institute, respectively - two organizations for which improving health was not the sole primary intent. 


\section{Main strengths and limitations}

We developed a clear, comprehensive and practical definition for the global health system that has the potential to facilitate consistent and in-depth research about this system in the future. In our study, the definition proved operational to map the structure of this system and analytically helpful for facilitating further understanding of the group of actors and their interconnections that people have come to naturally think of as the 'global health system'. While this study was designed to yield a map of actors, it did not select actors based on their power or influence; this means the results are limited in terms of what features are analyzed. Results may also overlook some of the most important entities. The validation exercise with key global health stakeholders in December 2014 confirmed these findings; stakeholders agreed the definition was analytically helpful and the consultation revealed that the derived mapping does not exactly match with prevailing expert views of the system. However, this article presents a first systematic mapping of the global health system that can be improved upon in future exercises. Importantly, the use of our definition combined with Internet searches allowed for the systematic compilation of the largest list of global health actors to date. While the algorithm underlying the Google query refinement "related:URL" is not publicly available, the search engine is publicly available which makes it possible to replicate the methodology. Notably, any replications of the methodology will yield results that reflect the relationship between web pages on the date it is carried out.

Interestingly, despite defining the global health system from a holistic, global perspective, results may suggest a global health system characterized predominantly by the development agenda. This may reflect the nature of the global health system as it currently stands, defined by years of responding to global health challenges through a development lens. Alternatively, the methodology may have captured a portion of the global health system that is disproportionately focused on development assistance, perhaps as a result of using WHO as the search's starting point. This could have occurred due to a tendency for WHO to provide links to its funders on its website. In the latter case, the post-2008 emergence of global health actors may have been underestimated, as these actors may be more likely to focus on global public goods and threats rather than development assistance to low- or middle-income countries. In this case, the search algorithm may not have found them to be related to actors identified earlier in the search.

By placing WHO at the centre of the system, this study used only one point of entry to probe the global health system. Using WHO's website as the starting point of the search may have biased the search in favour of those types of actors to which WHO's internal policies allow the organization to hyperlink. This may have increased the number of traditional and non-controversial actors identified, such as other UN entities and major WHO funders. However, our decision to start with WHO is justified by the widely acknowledged prominence and important role of this UN entity within the global health system. Other points of entry in approaching this exercise have their own advantages and disadvantages; for example, we could have begun the search with several global health actors perhaps those with the largest global health budgets - but any starting list would have created its own biases.

Two other sources of potential bias have been identified. The use of www.google.com as well as a proxy server located in the U.S. may have biased results towards actors located in the U.S. However, the U.S.-based version of Google was selected because this is the domain of the original search engine launched by Google and is thus considered its standard product on which other location-based Google search engines are based [25]. Also, the default language of the WHO's website is English. Since related search functions consider textbased analysis of websites' content as one input when determining if web pages are related, the initial search may have biased results against actors whose default websites are not in English. This would include many important bilateral development agencies (e.g., Norway's Norad) and global health actors in developing countries (e.g., Brazil's Fiocruz).

\section{Conclusions}

This study developed a new definition for the 'global health system, presented a novel methodology for populating it, and began to analyze the structure of the resulting system as well as the characteristics of its components. Consultation with senior leaders from seven key global health organizations confirmed the usefulness of this exercise and revealed a need for future mapping exercises to be more globally representative.

Future research should build on the experience of this study, finding ways to more thoroughly saturate the network of actors in the global health system. For example, future approaches may involve adapting the study's methodology by using multiple purposively selected organizations from a variety of countries, and initiating a related search using the national Google websites corresponding to their headquarters' locations. Internet-based findings could also be triangulated with information from political and expert opinion leaders in global health. This approach may yield more geographically neutral results as well as a list of actors that more comprehensively represents the offline global health system. Furthermore, study of the system's structure using social network analysis suggests promise for more in-depth research in this area. Future studies should 
16. Bastian M., Heymann S., Jacomy M. Gephi: an open source software for exploring and manipulating networks. International AAAI Conference on Weblogs and Social Media; 2009.

17. Freeman LC. Centrality in networks: I. Conceptual clarification. Soc Networks. 1979;1:215-39.

18. Oritz-Arroyo D. Discovering sets of key players in social networks. In: Abraham A, Hassanien A-E, Snasel V, editors. Computer communications and networks: trends, tools and research advances. London: Springer; 2010.

19. Hoffman SJ, Røttingen J-A, Bennett S, Lavis JN, Edge JS, Frenk J, et al. A review of conceptual barriers and opportunities facing health systems research to inform a strategy from the World Health Organization facing health systems research to inform a strategy from the World Health Organization. 2012.

20. World Health Organization. Hosted health partnerships: report by the secretariat. 2013.

21. The Lancet. What has the gates foundation done for global health? Lancet Elsevier Ltd; 2009;373(9675):1577. [cited 2014 Nov 15]. Available from: http:// www.ncbi.nlm.nih.gov/pubmed/19427940.

22. United Nations. UN at a Glance [Internet]. Available from: http://www.un. org/en/about-un/.

23. The World Bank. Archives: World Bank Historical Chronology: 1970-1979 [Internet]. Available from: http://siteresources.worldbank.org/EXTARCHIVES/ Resources/WB_Historical_Chronology_1944_2005.pdf.

24. Institute for Health Metrics and Evaluation. Financing Global Health 2013: transition in an age of austerity. Seattle, WA: IHME; 2014.

25. Google. How we started and where we are today. [Internet]. 1998. Available from: https://www.google.com/int//en/about/our-story/.

26. Acuto M. Diplomats in Crisis. Diplomacy and Statecraft. 2011;22(3):521-39.

27. McMaster Health Form. Health Systems Evidence: Advanced Search, website, McMaster Health Forum. http://www.healthsystemsevidence.org/ advanced-search.aspx. Accessed 27 Nov 2014

\section{Submit your next manuscript to BioMed Central} and we will help you at every step:

- We accept pre-submission inquiries

- Our selector tool helps you to find the most relevant journal

- We provide round the clock customer support

- Convenient online submission

- Thorough peer review

- Inclusion in PubMed and all major indexing services

- Maximum visibility for your research

Submit your manuscript at www.biomedcentral.com/submit 